\title{
Avoiding Buffer-Overloading in the Wireless Sensor Network
}

\author{
Rupali saini ${ }^{1}$ and Harsukhpreet Singh $^{2}$ \\ ${ }^{1}$ Dept. of computer science and engineering, CTITR, Jalandhar, India \\ ${ }^{2}$ Dept. of electronics and communication, CTITR, Jalandhar, India \\ ${ }^{1}$ saini.rupali33@yahoo.com, ${ }^{2}$ Harsukhpeet@gmail.com
}

\begin{abstract}
The main problem of wireless sensor network is congestion occur due to bufferoverloading. Due to this issue, the performances of the network such as network throughput and packet loss are affected. To reduce the buffer-overloading from the WSN a comparative study has been done between AODV and QAODV using queue length buffer-overloading equation. In this paper, we proposed QAODV using queue length buffer-overloading equation through which major cause of congestion, buffer-overloading is reduced from the network.
\end{abstract}

Keywords: WSN, QAODV, AODV, PDR

\section{Introduction}

A Mobile Ad-Hoc Network (MANET) is a momentary network; the mobile devices in an ad-hoc network are communicating through wireless links without any pre-existing infrastructure. Wireless sensor network is a network which transfers data from source to destination through intermediate nodes, it means that data transfer source to destination hop-by-hop. The one major problem of this network is congestion; it may take place inbetween nodes when data packets are traveling from source to destination. Congestion in a network may occurs when the incoming traffic is larger than the capacity of the network $[1,2,4]$. Congestion affected the overall performance of the network which is major problem of congestion. Congestion leads to packet losses and bandwidth degradation and wastes time and energy on congestion recovery $[13,6,7,5]$.

The main reason of congestion is buffer-overloading of the network due to which nodes start packet dropping. When the queue of the intermediate node of the network is exhausted then congestion occurs [14,5]. In such networks, packet retransmission occurs due to which throughput of the network decreases and all other performances of the network suffer. WSN is a multi-hope network due to which Performance of entire wireless network is affected if congestion occurs at a single node [12]. In this paper, we proposed QAODV (AODV using queue length equation) technique for avoiding the congestion's main cause buffer-overloading in WSN. By using queue length we proposed a network which gives better performance than AODV on the basis of throughput, packet loss, end-to-end delay, packet delivery ratio and normalized load. Congestion is an issue in the WSN due to which so many huddles comes in the network performance and these huddles are ;- packet loss, packet retransmission due to which more energy consumption of the source node. In this paper, we tried to avoiding the congestion and increase the lifetime of sensor nodes by reducing the requirement of retransmission of the packet. 


\section{Related Work}

Chen S. et al.[2005] proposed a congestion prevention scheme based on light burden buffer management. According to author this approach prevented the data packet loss due to overflowing the buffer of sensor node because it automatically accepts the sensor forwarding rate to nearly optimal without causing congestion [5].

Parul K. et al.[2010] presented a comparative analysis between AODV, DSDV, LEACH for congestion control on WSN. In this paper, author observes the performance of these three protocols and concluded that AODV gives better performance than other two protocols [10].

Samundiswary P. et al.[2010] proposed a protocol S-AODV for decreasing the routing overhead of AODV on the network. In this paper, author describes the working of AODV and compares its performance with his proposed protocol [9].

Anju G. et al.[2010] presented a comparative analysis of three routing protocol of WSN like AODV, DSDV, TORA. Author conclude from the results that AODV gives overall better performance than other two protocols [8].

Chatterjee A. et al. [2012] proposed a transport protocol using single hop cluster based tree topology for congestion prevention in wireless sensor network. In this protocol, there is one cluster-head in every cluster and every cluster-head is the parent of other clusterhead due to which the whole network arranged in the form of a tree. Data received and send by the cluster-head in the network which leads to congestion avoidance and less energy consumption in wireless sensor network [11].

Senthil K, et al. [2012] presented a technique for determining the congestion status of the each node in the network. In this paper describe the queue length equation for preventing network gets congested due to buffer -overflow [12]

Devi M. et al.[2013]presented different techniques for avoiding congestion. In this paper, author determines congestion status and sent a message to a source if congestion present on the link [13].

Charalambos S. et al. [2014] proposed an algorithm for controlling the congestion in wireless sensor network the algorithm is called hybrid algorithm. In Hybrid algorithm both wireless control method traffic control and resource control used in the network. One method is applied to the network according to the network condition and according to its need due to which lifetime, as well as throughput of the network, is increased [14].

\section{Techniques for Controlling Congestion}

There are many ways to reduce congestion on wireless sensor network. By applying some algorithm or technique congestion can be reduced from the wireless sensor network. AODV is a protocol which gives the overall best performance from rest of the protocol like DSR, DSDV, TORA $[9,10,16]$. The performance of AODV can be modifying by applying congestion reducing algorithm or equation on a protocol in the wireless sensor network. In this paper we apply an average queue length buffer- overflow equation on AODV i.e. QAODV for removing queue-overflow problem due to which congestion occurs in the wireless sensor network and compare its results with AODV $[9,10,11]$.

AODV (Ad-hoc On-demand Distance Vector) is the protocol which permits nodes to transfer data to another node those are not directly connected with each other. It is better than other protocols because it selects the shortest and less congestion path between source and destination for transferring data. Before establishing the path between source and destination, it sends a broadcast message source to destination that is called Route Request Broad Cast Flood shown in fig no. 1. Destination sends a unicast message to the source after receiving a broadcast message that is called Route Reply Propagation shown in fig no. 2. After receiving route reply message by source a path is selected by the network which having minimum intermediate nodes and less congestion [9]. Due to these 
functions AODV having more ability for reducing congestion from rest of protocol and we are applying queue length equation on AODV for enhancing its performance.

\subsection{Implementation of Average Queue Length Equation on AODV(QAODV)}

In wireless sensor network, the major reason of congestion is buffer overloading of the intermediate nodes of selected path. Networks unawareness about the buffer capacity of the selected path intermediate nodes is the cause of congestion. When the network starts transferring data through that nodes which having less space in its buffer then it starts dropping packet due to which throughput of the network decreases. It means to avoid buffer overloading, the network should aware about the capacity of the buffer of every node. This can be done by using average queue length technique. The average queue length purpose is gathering the entire traffic variations and follows the long-term variations of the instant queue that duplicates the stable network congestion [13]. The equation is defined as:-

$\mathrm{L}_{\mathrm{q}} \quad\left(1-\mathrm{W}_{\mathrm{q}}\right) \quad * \quad \mathrm{~L}_{\mathrm{q}}+\mathrm{L}_{\mathrm{iq}} \mathrm{W}_{\mathrm{q}}$

Where, $\mathrm{L}_{\mathrm{q}}=$ Instantaneous queue length

$\mathrm{W}_{\mathrm{q}}=$ weight factor that helps in regulating the congestion in the network.

Average queue length equation determines the number of resources or buffer space available to each link to the network that is called prediction value. Which link having the minimum prediction value from source to destination that link will be selected for data transfer shown in fig no. 3 . Red line arrow shows the selected path because the sum of the prediction value of this link is a low prediction as compare to another link. In this way, the network can avoid buffer overloading. This equation reduces the chances of bufferoverflowing in the network. Due to this, we can control congestion problem and packetlost from the WSN [12] [13]. In this paper we applied average queue length equation on AODV (QAODV) for reducing congestion from WSN for enhancing AODV performance.

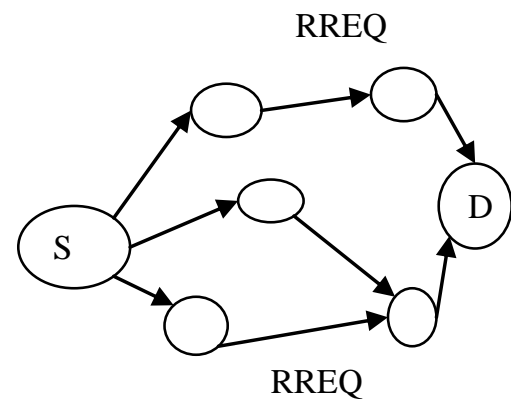

Figure 1. Route Request Message

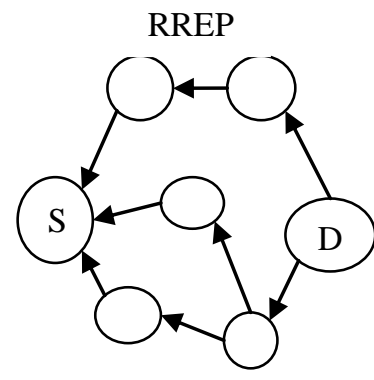

RREP

Figure 2. Route Reply Message 


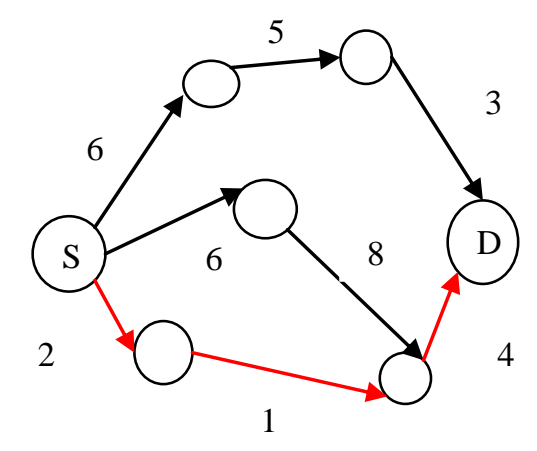

Figure 3. Prediction Value of Link

\section{Flow Chart of Work Done}

The flow chart of the proposed work is shown in fig. 4. Firstly we deployed sensor nodes in the fixed area shown in fig. 5. Then implement the ad-hoc -on demand distance vector routing protocol on the wireless sensor network (AODV). Average queue length equation is implementing on AODV for enhancing the performance of AODV and reducing congestion as well as buffer overflow problem from the network. By using the average queue length equation prediction value of each node and link is determined for transferring data source to destination. Then network select the link from source to destination which having smallest prediction value. It means network select that path for transferring data which have buffer space than other available path and least probability of network. After this, we compared the result of AODV and AODV with average queue length equation (QAODV) for analyzing the performance of both techniques. By using these following steps our proposed work for reducing congestion is completed. 


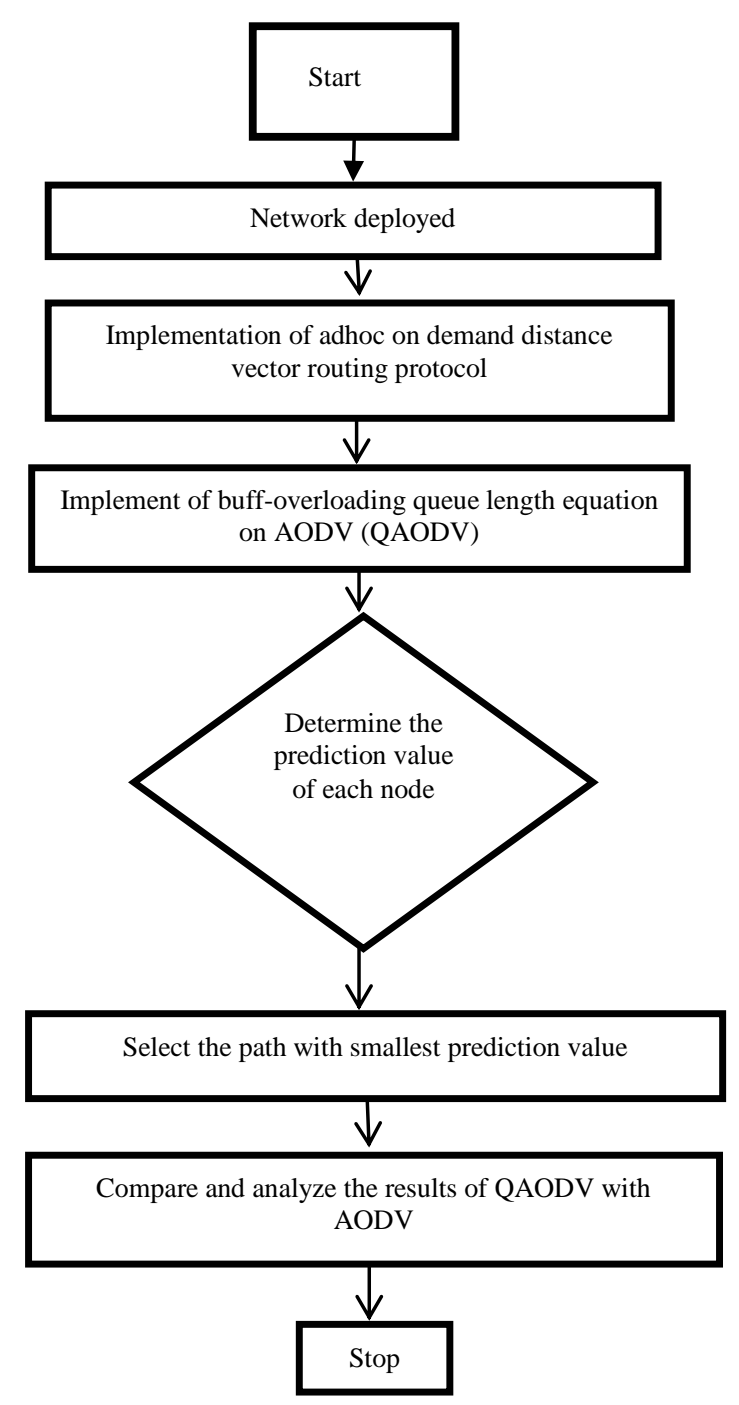

Figure 4. Work Flow Chart

\section{Simulation and Results}

\subsection{Simulation Environment}

We implemented our work on NS2.35. It is a network simulator which supports simulations of TCP and UDP and also supports multicast protocol over wired and wireless network and MAC layer protocols etc. Five parameters have been considered and tested in the simulation, ]version 2.35 with the wireless package. In all five test parameter, sink and all other sensor nodes are stationary. Twenty-four nodes are uniformly deployed on area $800 \times 800$, whose frequency and network range is $2.4 \mathrm{Ghz}$ and 18 meters respectively. Performance evaluation of two congestion avoidance methods AODV and QAODV is conducted on following metrics captured from NS2.35 statistics:- Network throughput, total packet loss, network end-to-end delay, packet delivery ratio, normalized network load. We implemented wireless sensor nodes in fixed area shown in fig. 5.Then we implemented AODV on NS2 in the wireless sensor network in which source node broadcast request message and destination send reply message shown in fig. 6,7 respectively and observe congestion in the network shown in the simulation fig. 8 . 
We also implement the average queue length equation on AODV in the wireless sensor network which determines prediction value of each node shown in fig. 9.

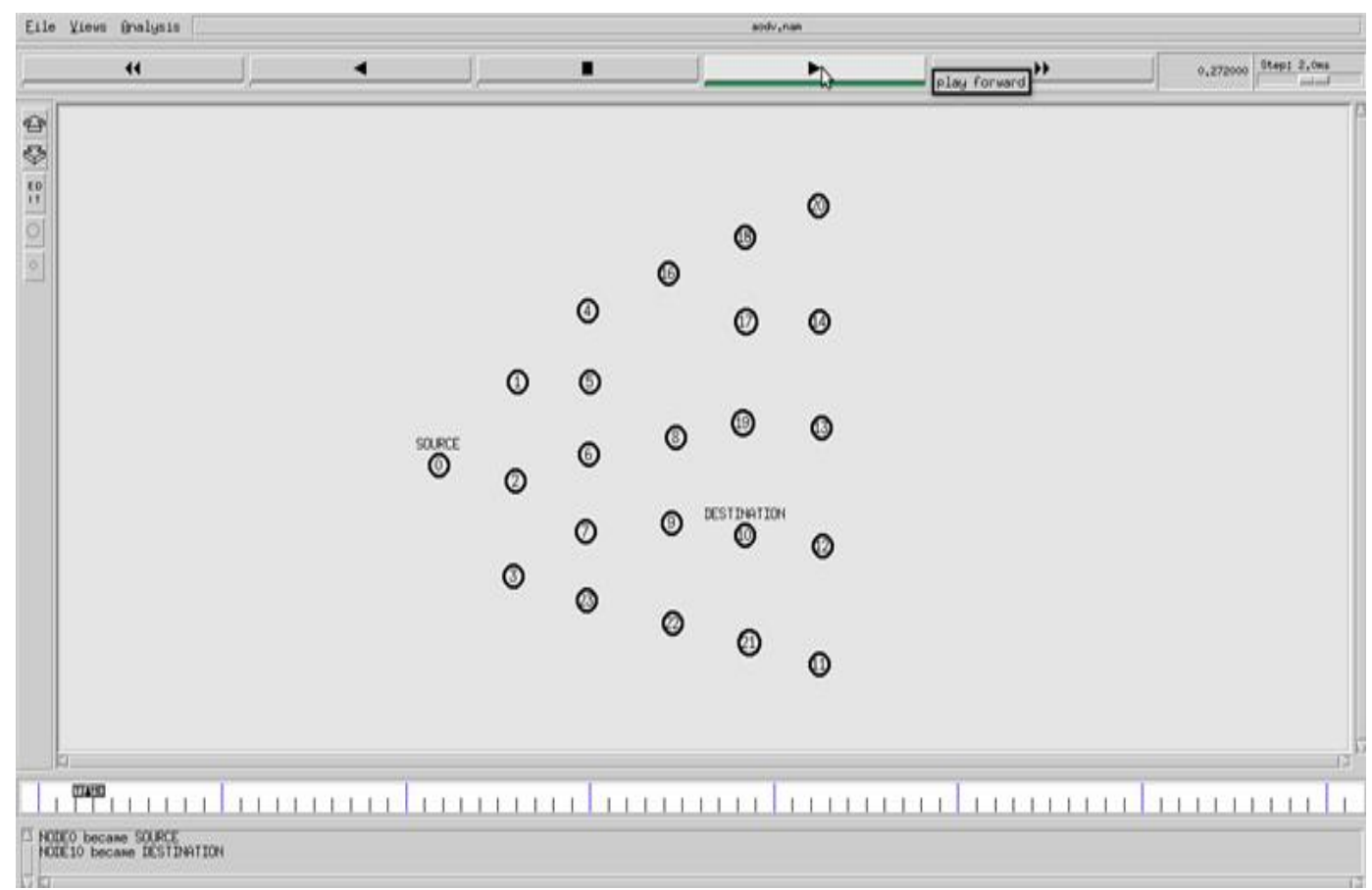

Figure 5. Simulation Snapshot of Sensor Nodes Deployed

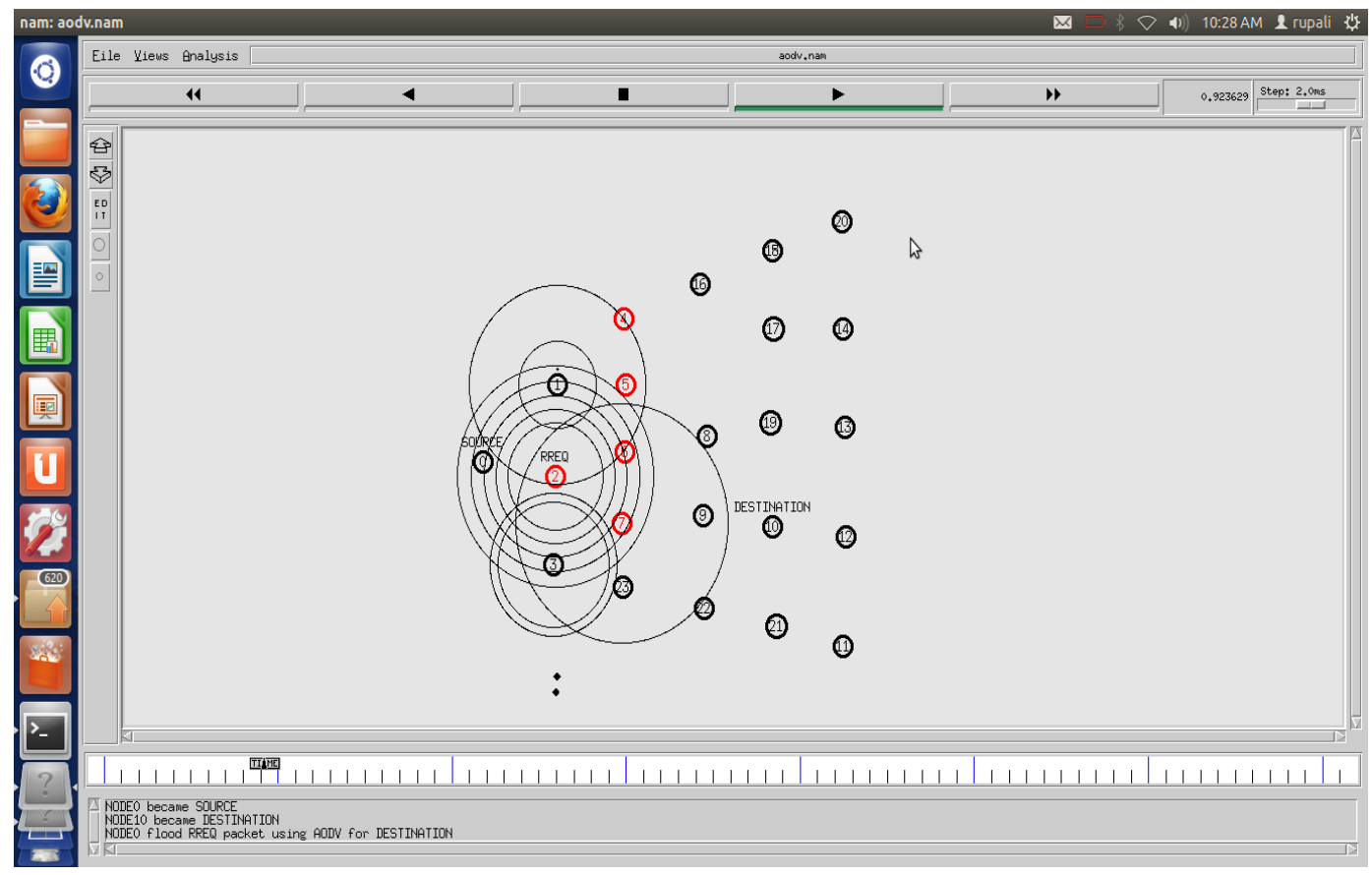

Figure 6. Simulation Snapshot of Broad Cast Request Message of AODV 


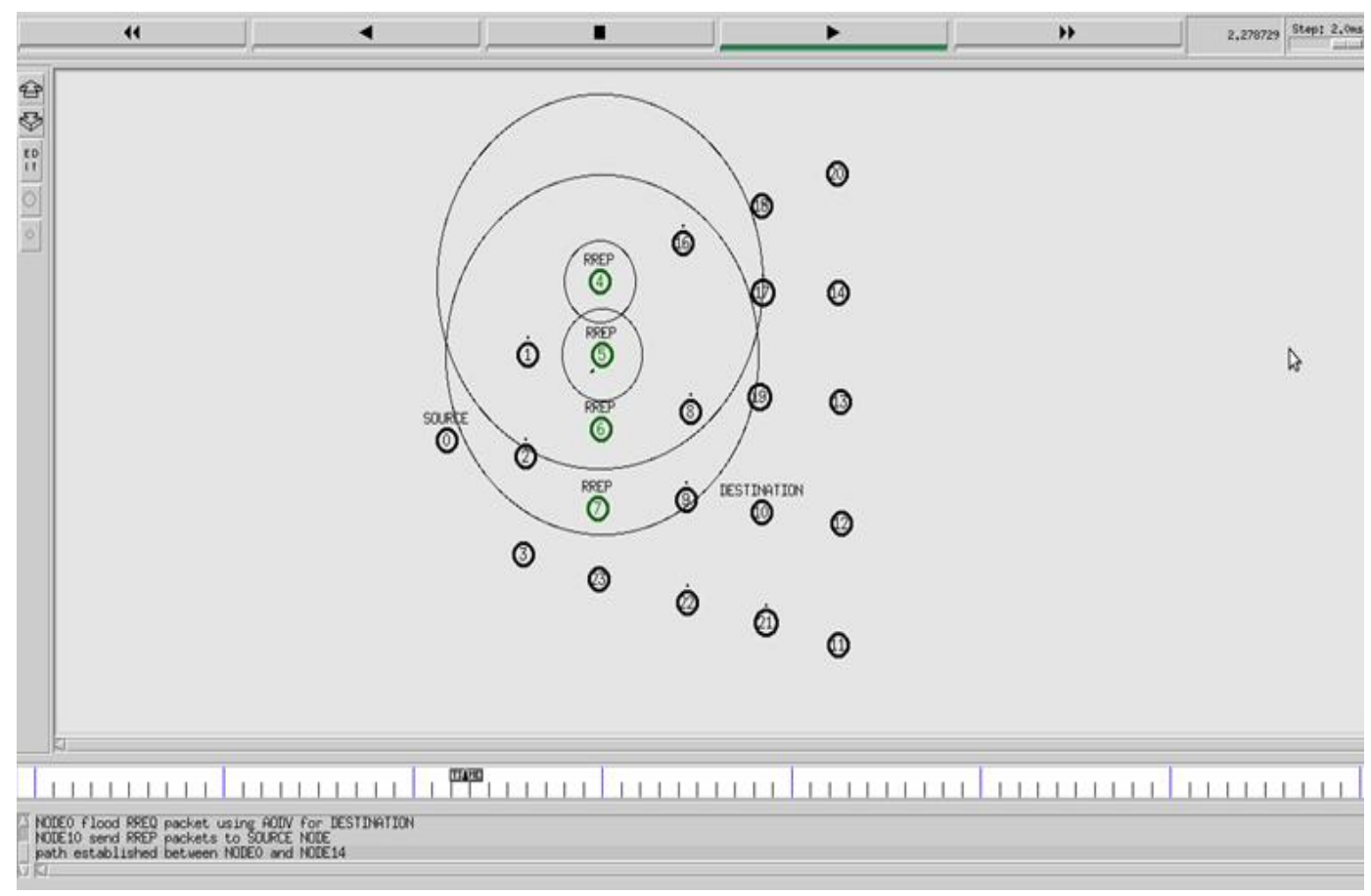

Figure 7. Simulation Snapshot of Reply Message by Sink of AODV

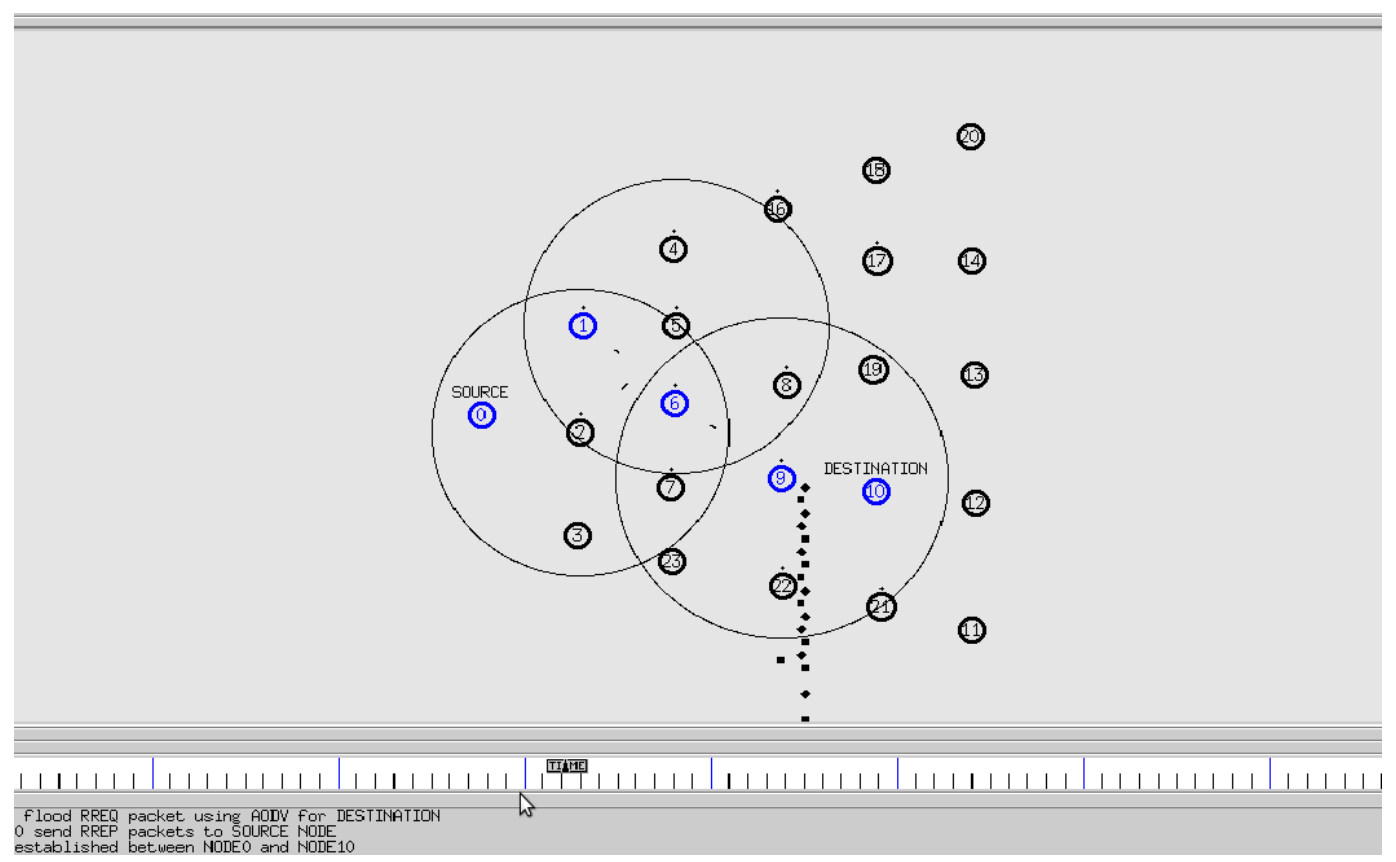

Figure 8. Simulation Snapshot of Congestion in AODV 


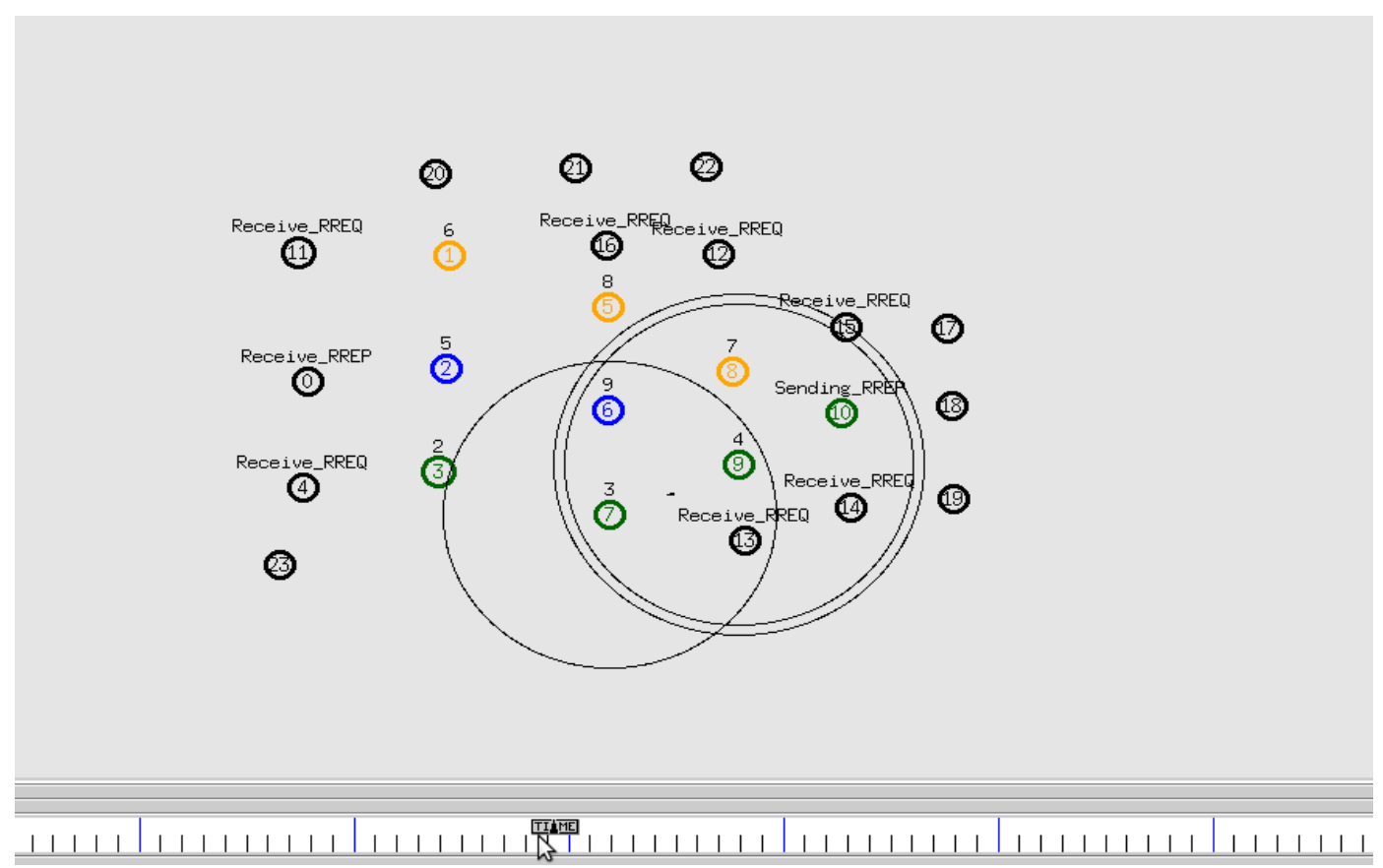

Figure 9. Simulation Snapshot of Prediction Value of QAODV

\subsection{Results}

The graphical result shows the large performance difference between AODV and QAODV. Results are evaluated on the basis of the different scenario.

Throughput:- In the first scenario, we plot the throughput graph. By studying the fig. 10 , we noticed that throughput of the QAODV is higher than AODV in the WSN. It means average queue length buffer-overflowing algorithm improve the AODV. QAODV prevents buffer-overflow due to which throughput of the network increase per second. It requires more time for path selection than AODV but increasing the throughput of the network without losing data.

Packet-loss:- In the second scenario, we plot packet-loss graph in the network. The graphical result of fig. 11 shows that QAODV having lesser packet-loss as compared to AODV. It means AODV having packet-loss as compare to QAOD during the data transmission on the WSN. QAODV determine the maximum resource available on the links from source to the destination due to which by using QAODV packet-loss reduces from the network. Packet-loss of network is decreased effectively in the WSN when we apply average queue length on AODV as compare to AODV only.

Packet delivery ratio:- We plot the PDR graph of the sink by using AODV and QAODV in the third scenario. By studying the graphical result of fig. 12, we noticed that QAODV gives higher packet delivery ratio (PDR) than AODV. QAODV improves the performance of AODV by finding the prediction value of the link due to which value of PDR increases in the network.

End- to- end delay:- In the fourth scenario, we plot the end-to-end delay graph of the sink by using AODV and QAODV. By analyzing the result of fig. 13, we notice that endto- end delay of QAODV is very lower than AODV. It means AODV having the high delay in the packet delivery because congestion arises when buffer start overflow of selected path due which delay increases in packet delivery. QAODV prevent bufferoverflow and congestion on the network by selecting maximum resource available on the path.

Normalized Routing load:- In the last scenario we plot graph normalized routing load graph of the sinks by using AODV and QAODV. We noticed in fig. 14, that normalized 
load of the network is very lesser in the case of QAODV as compare to AODV. QAODV having less load than AODV because once it determines prediction values then no need to send a message again and again for finding a path.

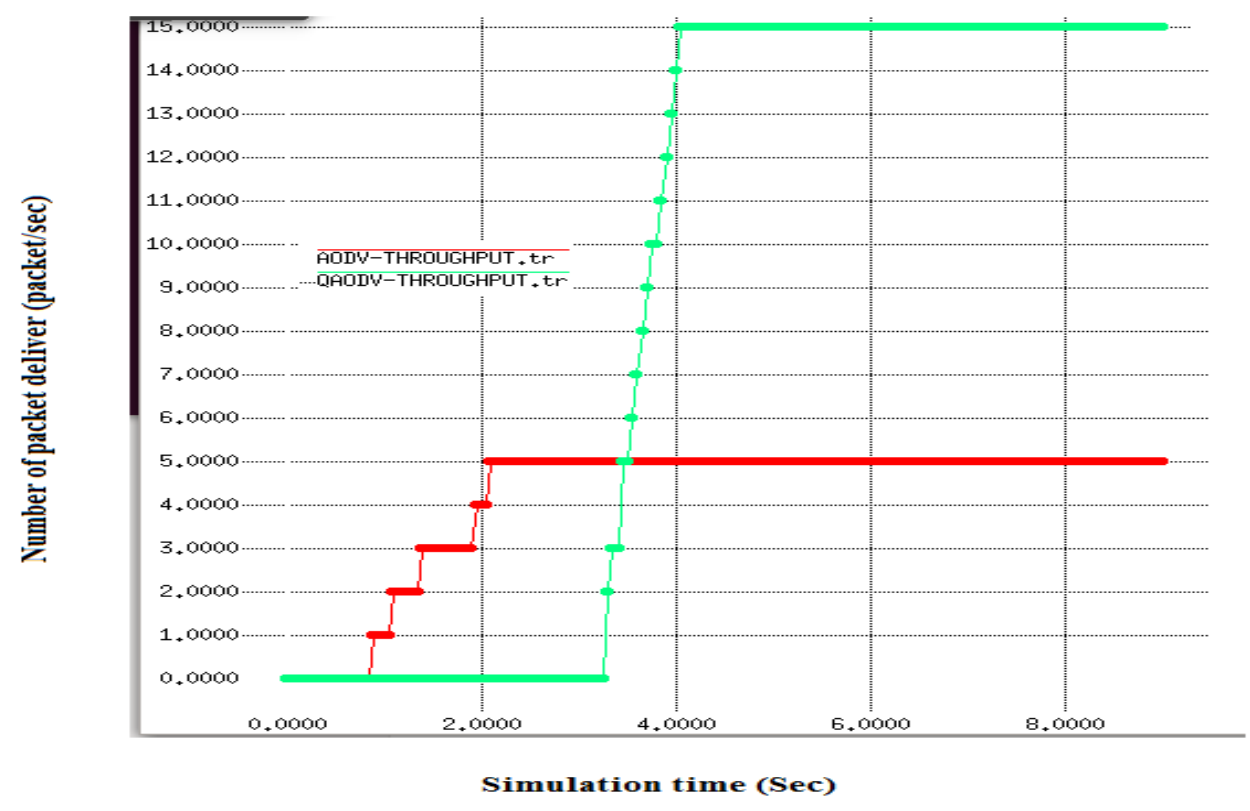

Figure 10. Throughput Graph

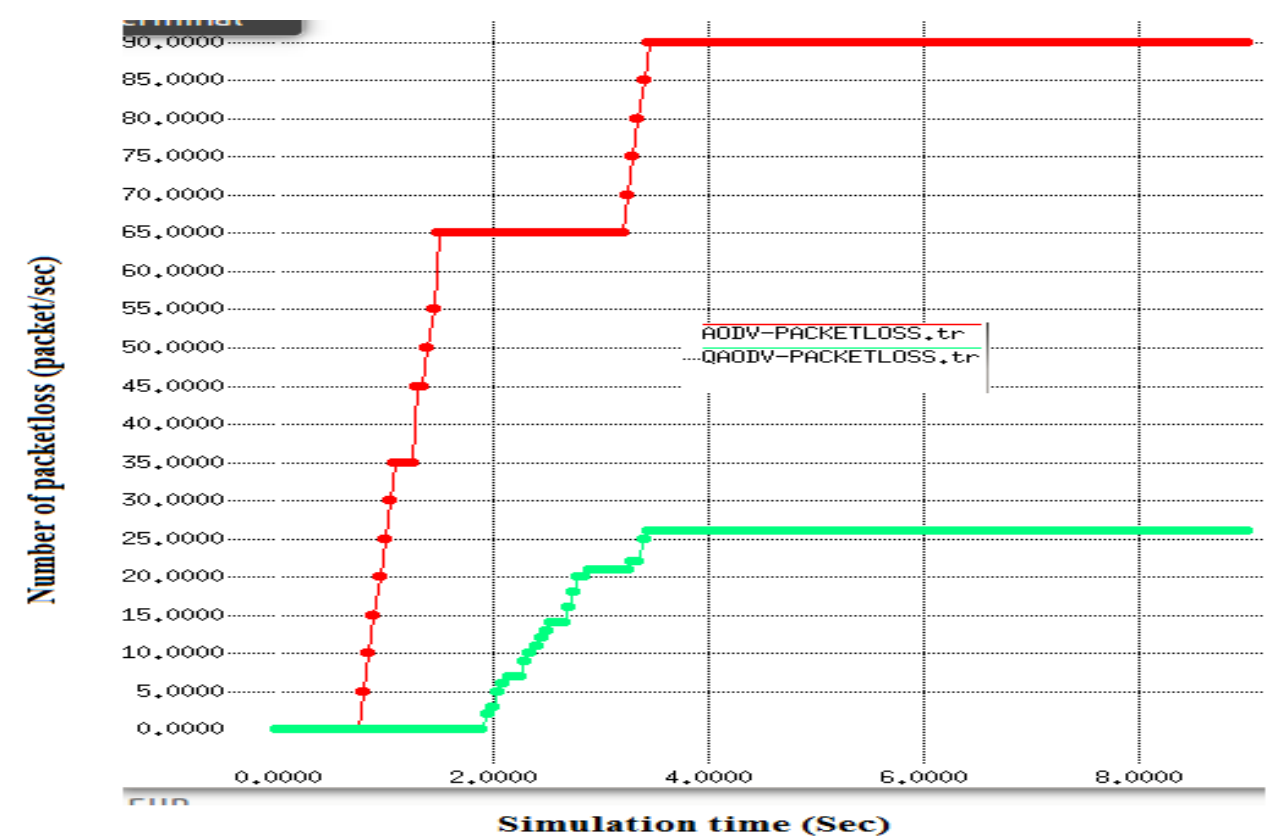

Figure 11. Packet-loss Graph 


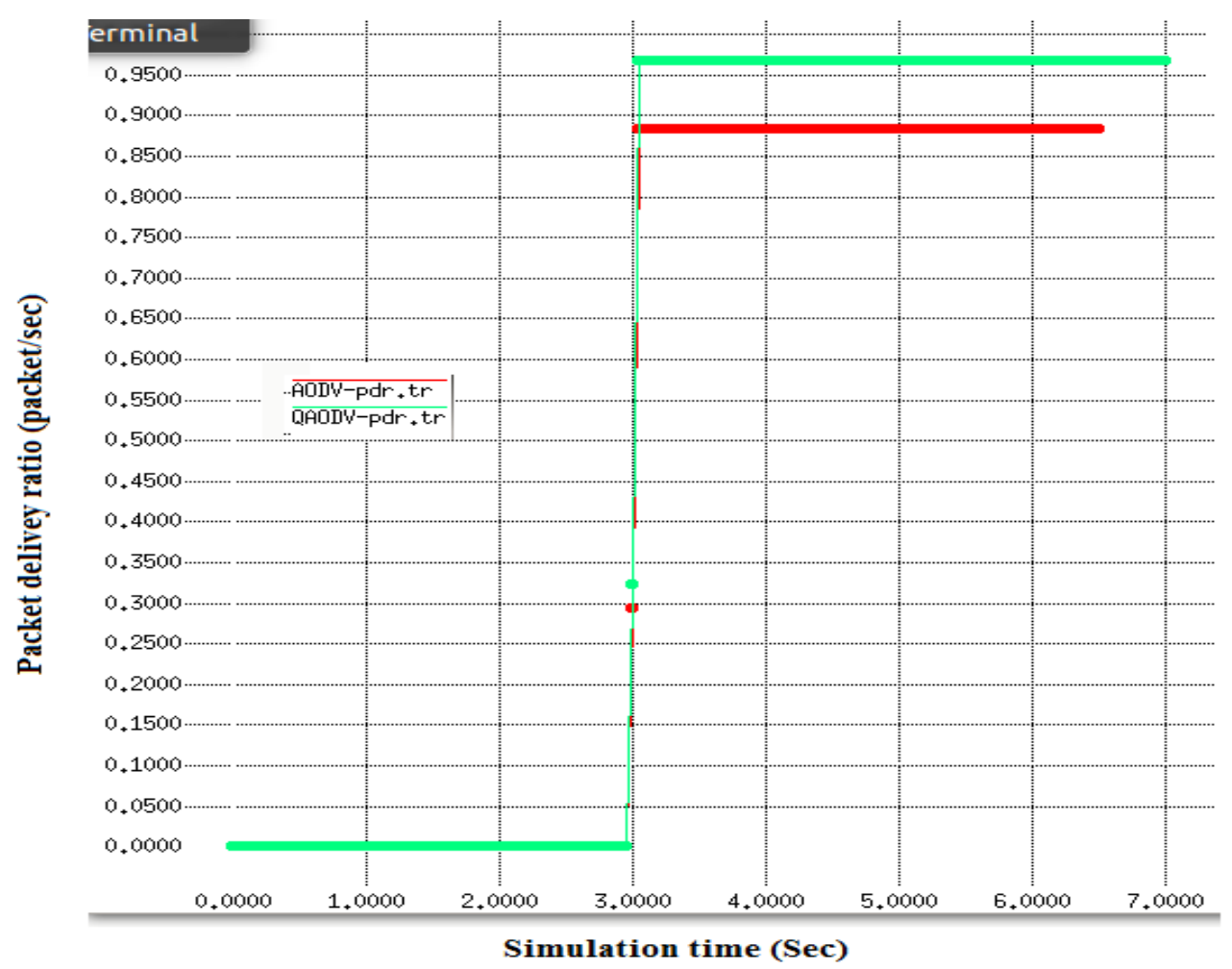

Figure 12. PDR Graph

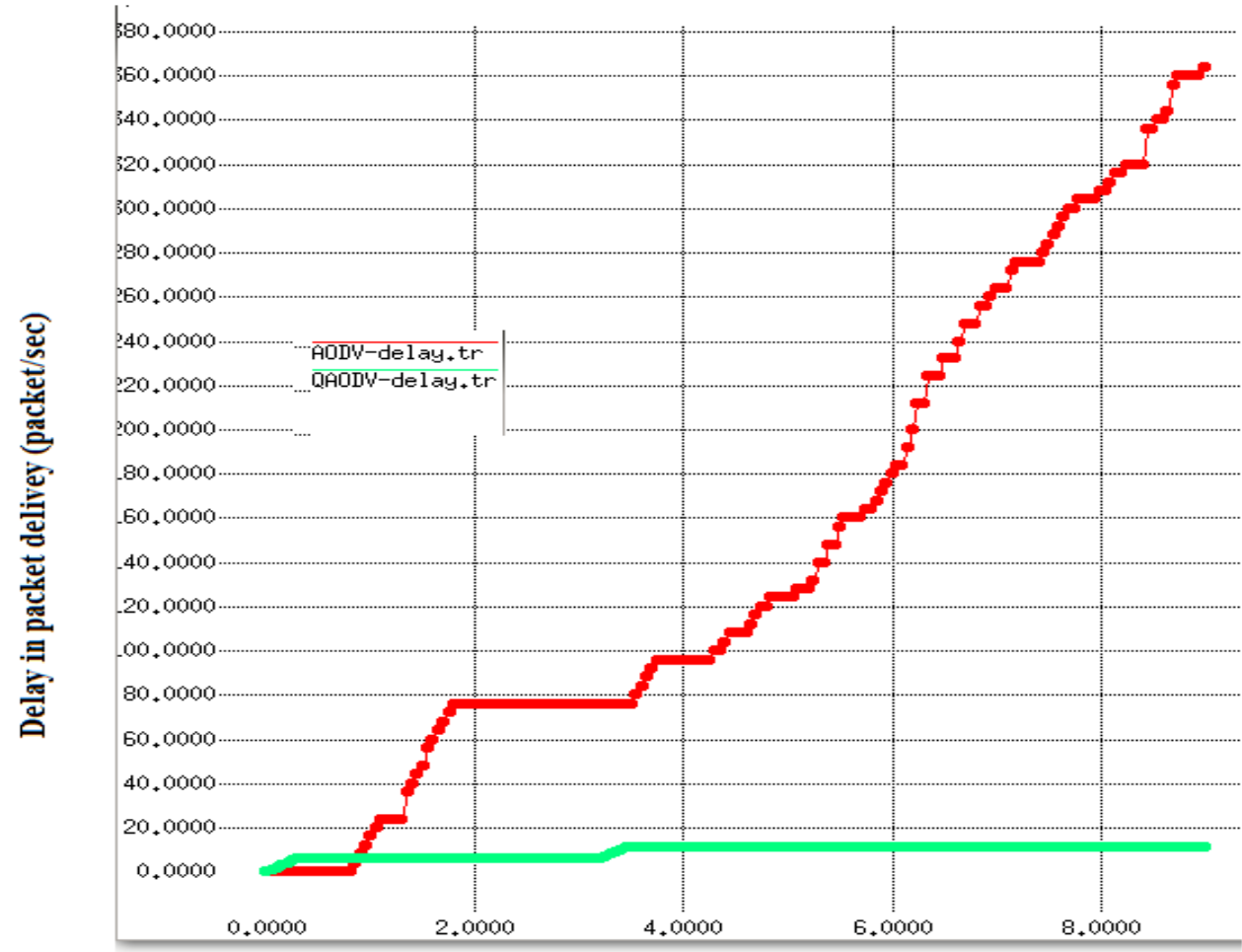

Simulation time (Sec)

Figure 13. Delay Graph 


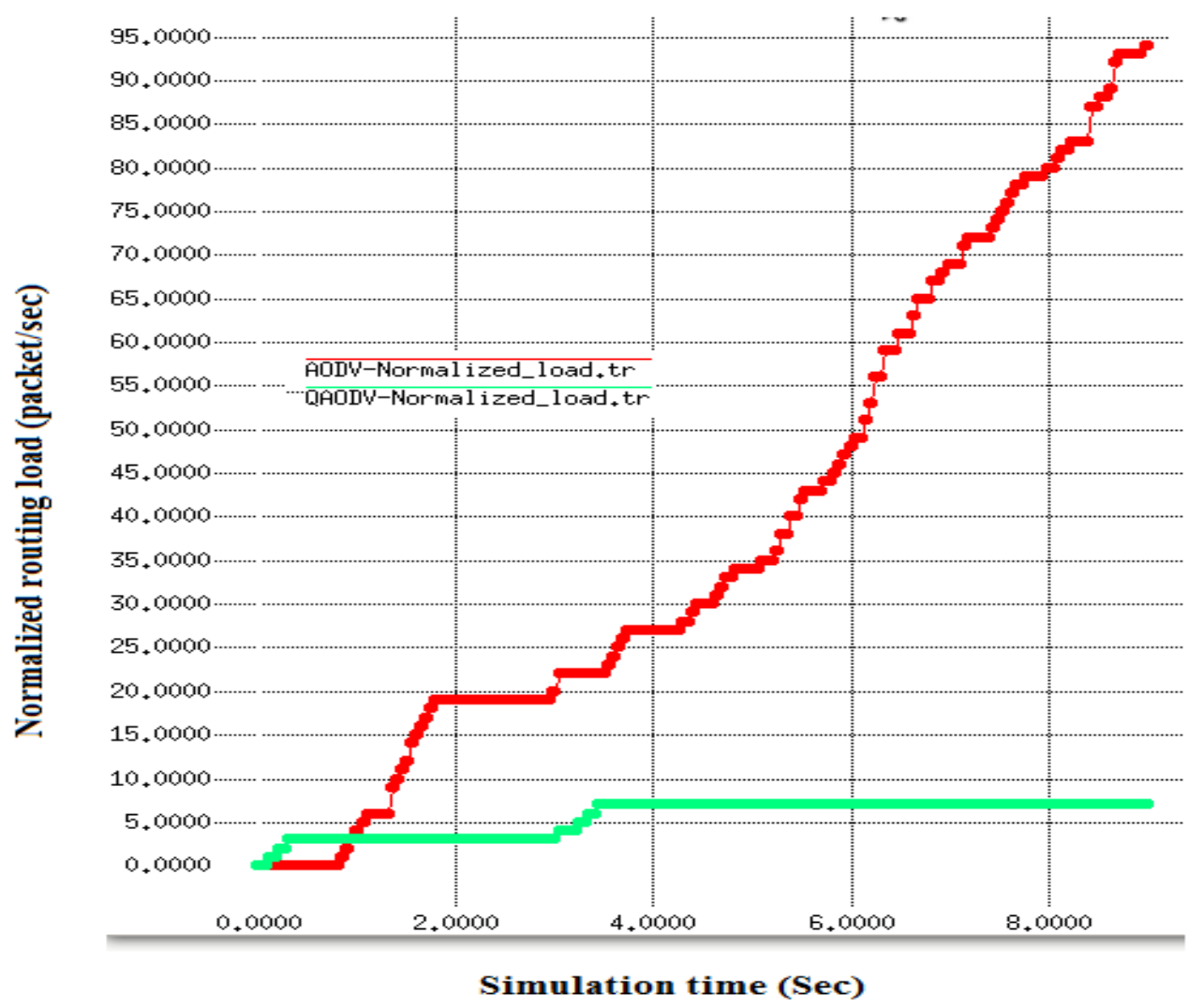

Figure 14. Normalized Routing Load

\section{Conclusion}

QAODV is proposed for avoiding the congestion major cause buffer-overloading. AODV select the less congestive path on the basis of traffic on the link and QAODV select the path on the basis of resources availability on the link. AODV and QAODV both are techniques for congestion avoidance but when we join AODV with buffer-overflow algorithm, it gives good results. We conclude from the result shown above that QAODV enhance the performance of AODV by avoiding the buffer-overloading from the network and select the better path than AODV.

\section{References}

[1] A. Gupta, "Karnaugh Maps An Artificial Intelligence Approach", IEEE, (1990), pp. 12-13.

[2] J. N. Al Karaki and A. E. Kamal, "Routing techniques for wireless sensor network", IEEE, vol. 11, no. 6, (2004), pp. 6-28.

[3] Y. G. Iyer, S. Gandham and S. Venkatesan, "A Generic Transpot Layer Protocol For Wireless Sensor Network", IEEE, (2005), pp. 449-454.

[4] O. B. Akan and F. Akyildiz, "Event-To-Sink Reliable Transport In Wireless Sensor Network", IEEE, vol. 13, no. 5, (2005), pp. 1003-1016.

[5] S.-G. Chen and A. Yang, "Congestion Avoidance Based On Light-Weight Buffer Management In Sensor Network", IEEE, vol. 17, no. 9, (2006), pp. 934-946.

[6] G. Rajsekar, M. K. Mathew, N. Dineshra, S. R. Barath, M. N. Sudha and M. L. Valarmathi, "Collision Avoidance Schem In Energy Constrained Wireless Sensor Network Using MAC Protocol”, IEEE, (2008), pp. 1-4.

[7] L.-Q. Tao, "Enhanced Congestion Detection And Avoidance For Multiple Class Of Traffic In Sensor Network", IEEE, (2009), pp. 726-730.

[8] A. K. Gupta, H. Sadawarti and A. K. Verma, "Performance Analysis Of AODV, DSR, TORA Routing Protocol", International Journal Of Engineering And Technology, vol. 2, no. 2, (2010), pp. 226-231.

[9] P. Samundiswary and P. Danajayan, "Performance Analysis Trust Based AODV For Wireless Sensor Network", International journal of computer application, vol. 4, Number 12, 2010, pp. 6-13. 
[10] Parul kansal, Deepali Kansal, Arun Balodi, "Comparison Of Various Routing Protol In Wireless Sensor Network," International journal of computer application, vol. 5, no. 11, (2010), pp. 14-19.

[11] A. Chatterjee, A. K. Mukhopadhyay and D. Mukherjee, "The Transport Protocol For Congestion Avoidance In Wireless Sensor Network", IEEE, (2012), pp. 389-393.

[12] N. Bobadea and N. Mhala, "Performance evaluation of AODV and DSR on-demand routing protocols with varying MANET size", Proceeding of International Journal of Wireless \& Mobile Networks, (2012).

[13] S. kumaran and V. Shankarnarayanan, "Congestion Free Routing In Adhoc Network", Journal of computer science, (2012), pp. 971-977.

[14] M. Devi and V. R. Uthariaraj, "Congestion Based Routing Recovery Technique For Manet", Journal of theoretical and applied information technology, vol. 54, (2013), pp. 74-81.

[15] C. Sergiou, V. Vassiliou and A. Paphitis, "Hierarchial Tree Alternative Path Algorithm For Congestion Control In Wireless sensor network", ELSVIER, vol. 11, no. 1, (2013), pp. 257-272.

[16] C. Sergiou and V. Vassiliou, "A Hybrid Algorithm For Efficient Congestion Control In Wireless Network", IEEE, (2014), pp. 1-5.

[17] S. Mitra and A. De Sarkar, "Energy Aware Fault Tolerant Framework In Wireless Sensor Network", IEEE, (2014), pp. 139-145.

[18] R. Dasgupta, R. Mukherjee and A. Gupta, "Congestion Avoidance Topology In Wireless Sensor Network Using Karnaugh Map”, IEEE, (2015), pp. 89-96.

[19] R. Kumar, "Analysis and enhancement of adaptive packet techniquie in wireless sensor network", Inter National Journal Of Engineering Trend And Technology, vol. 24, no. 5, (2015), pp. 227-230.

[20] T. Shrma, H. Singh and A. Sharma, "A Comparative Review On Routing Protocol In Wireless Sensor Network”, International Journal Of Computer Application, vol. 123, no. 14, (2015), pp. 28-33. 